\title{
ON AN EXTENSION OF HÖLDER'S INEQUALITY
}

\author{
C.E.M. Pearce and J. Pečarić
}

An new extension of Hölder's inequality is derived. This is shown to follow from a generalisation of Steffensen's inequality.

\section{INTRODUCTION}

An extension of Hölder's inequality for monotonic $n$-tuples has been given by Mudholkar, Freimer and Subbaiah [4]. Iwamoto, Tomkins and Wang [2] have presented this result for infinite sequences and given a corresponding integral analogue, Theorem A below. For the purpose of enunciating this analogue, we first present the following lemma from Freimer and Mudholkar [1].

LEMMA 1. Let $b$ be a continuous, positive, nonincreasing and integrable function on $[0, \infty)$ and $M$ a positive, real number. Then there exists a number $K, 0 \leqslant K<M$, such that

$$
b(K) \leqslant \int_{K}^{\infty} b(t) d t /(M-K) .
$$

The integral result of Iwamoto, Tomkins and Wang is now as follows.

Theorem A. Let $b, M, K$ be as in Lemma 1. Then

$$
\int_{0}^{\infty} a(t) b(t) d t \leqslant\left(\int_{0}^{M} a(t)^{p} d t\right)^{1 / p}\left(\int_{0}^{M} \widehat{b}(t)^{q} d t\right)^{1 / q}
$$

for every nonincreasing, differentiable function $a$ on $[0, \infty)$ and $p>1$, where $p^{-1}+$ $q^{-1}=1$ and

$$
\widehat{b}(t)=\left\{\begin{array}{ll}
b(t), & 0 \leqslant t<K \\
\int_{K}^{\infty} b(t) d t /(M-K) & K \leqslant t \leqslant M
\end{array} .\right.
$$

The inequality in (1) is reversed if $p<1$ and $a$ is a nondecreasing, differentiable function. In both cases, equality holds if $a(t)^{p}=c \hat{b}(t)^{q}, 0 \leqslant t \leqslant M$ (where $c$ is a constant) and $a(t)=a(K), t \geqslant K$.

In this paper we give a very simple proof of a more general result.

Received 11th July, 1994

Copyright Clearance Centre, Inc. Serial-fee code: 0004-9729/95 \$A2.00+0.00. 
Theorem A appears to exhibit some connection with the well-known Steffensen inequality for a monotonic function. So a natural question is whether the Steffensen inequality or any of its generalisations can be used in the proof of the above result. The answer is in the affirmative. We use the following generalisation of Steffensen's inequality (see Mitrinović and Pečarić [3]).

Theorem B .

(i) Suppose that $f$ and $g$ are integrable functions on $[a, b], f$ is nonincreasing and $\lambda>0$. If a positive function $g$ satisfies the condition

$$
\lambda \int_{a}^{x} g(t) d t \leqslant(x-a) \int_{a}^{b} g(t) d t
$$

for each $x \in[a, b]$, then

$$
\frac{\int_{a}^{b} f(t) g(t) d t}{\int_{a}^{b} g(t) d t} \leqslant \lambda^{-1} \int_{a}^{a+\lambda} f(t) d t
$$

while if a positive function $g$ satisfies

$$
\lambda \int_{x}^{b} g(t) d t \leqslant(b-x) \int_{a}^{b} g(t) d t
$$

for each $x \in[a, b]$, then

$$
\lambda^{-1} \int_{b-\lambda}^{b} f(t) d t \leqslant \frac{\int_{a}^{b} f(t) g(t) d t}{\int_{a}^{b} g(t) d t} .
$$

In either case equality holds if $f$ is constant.

(ii) If $f$ is nondecreasing, the reverse inequalities hold in (3) and (5).

\section{Results}

Our main result is as follows.

ThEOREM 1. Let $f$ and $g$ be two integrable and positive functions defined on $[a, b]$ and let $M, K$ be real numbers satisfying $a \leqslant K<M \leqslant b$.

(i) Suppose that for every $x \in[K, b]$ we have

$$
\frac{1}{x-K} \int_{K}^{x} g(t) d t \leqslant \frac{1}{M-K} \int_{K}^{b} g(t) d t
$$

that $p>1, p^{-1}+q^{-1}=1$ and that $f$ is nonincreasing. Then

$$
\int_{a}^{b} f(t) g(t) d t \leqslant\left(\int_{a}^{M} f(t)^{p} d t\right)^{1 / p}\left(\int_{a}^{M} \widehat{g}(t)^{q} d t\right)^{1 / q}
$$


where

$$
\widehat{g}(t)=\left\{\begin{array}{ll}
g(t), & a \leqslant t<K, \\
\int_{K}^{b} g(t) d t /(M-K) & K \leqslant t \leqslant M
\end{array} .\right.
$$

The inequality in (7) is reversed if $p<1$ and $f$ is a nondecreasing function. In both cases, equality holds in (7) if

$$
f(t)^{p}=c \hat{g}(t)^{q} \quad a \leqslant t \leqslant M
$$

(where $c$ is constant) and

$$
f(t)=f(K) \quad t \in[K, b] .
$$

(ii) Suppose that for every $x \in[a, M]$ we have

$$
\frac{1}{M-x} \int_{x}^{M} g(t) d t \leqslant \frac{1}{M-K} \int_{a}^{M} g(t) d t
$$

that $p>1, p^{-1}+q^{-1}=1$ and that $f$ is nondecreasing. Then

$$
\int_{a}^{b} f(t) g(t) d t \leqslant\left(\int_{K}^{b} f(t)^{p} d t\right)^{1 / p}\left(\int_{K}^{b} \widehat{g}(t)^{q} d t\right)^{1 / q}
$$

where

$$
\widehat{g}(t)=\left\{\begin{array}{ll}
\int_{a}^{M} g(t) d t /(M-K) & K \leqslant t \leqslant M \\
g(t), & M<t \leqslant b
\end{array} .\right.
$$

The inequality in (9) is reversed if $p<1$ and $f$ is a nonincreasing function. In both cases, equality holds in (9) if

$$
f(t)^{p}=c \widehat{g}(t)^{q} \quad(K \leqslant t \leqslant b)
$$

(where $c$ is constant) and

$$
f(t)=f(M) \quad t \in[a, M]
$$

\section{Proof:}

(i) By (6), condition (2) is satisfied with $\lambda=M-K$ and $a$ replaced by $K$. Hence by Theorem $B(i)(3)$ holds, that is,

$$
\begin{aligned}
\int_{K}^{b} f(t) g(t) d t & \leqslant(M-K)^{-1} \int_{K}^{b} g(t) d t \int_{K}^{M} f(t) d t \\
& =\int_{K}^{M} f(t) \widehat{g}(t) d t .
\end{aligned}
$$


Also by definition

$$
\int_{a}^{K} f(t) g(t) d t=\int_{a}^{K} f(t) \widehat{g}(t) d t
$$

On addition we derive

$$
\int_{a}^{b} f(t) g(t) d t \leqslant \int_{a}^{M} f(t) \widehat{g}(t) d t .
$$

Relation (7) now follows from the Hölder inequality.

(ii) With $M$ in place of $b,(4)$ is satisfied with $\lambda=M-K$. As $f$ is nondecreasing, we have by Theorem $B(i i)$ that (5) holds with the inequality reversed, that is,

$$
\begin{aligned}
\int_{a}^{M} f(t) g(t) d t & \leqslant(M-K)^{-1} \int_{a}^{M} g(t) d t \int_{K}^{M} f(t) d t \\
& =\int_{K}^{M} f(t) \widehat{g}(t) d t .
\end{aligned}
$$

Further, by definition,

$$
\int_{M}^{b} f(t) g(t) d t=\int_{M}^{b} f(t) \widehat{g}(t) d t
$$

so that addition provides

$$
\int_{a}^{b} f(t) g(t) d t \leqslant \int_{K}^{b} f(t) \widehat{g}(t) d t .
$$

Relation (9) follows by the Hölder inequality.

The other cases follow similarly, while the statement for equality follows from the conditions for equality in Steffensen's and Hölder's inequalities.

Corollary 1.

(i) Suppose the assumptions of Theorem 1(i) are satisfied and further $g$ is nonincreasing. Then Theorem 1(i) is also valid if condition (6) is replaced by

$$
g(K) \leqslant(M-K)^{-1} \int_{K}^{b} g(t) d t .
$$

(ii) Suppose the assumptions of Theorem 1(ii) are satisfied and further $g$ is nondecreasing. Then Theorem 1(ii) is also valid if condition (8) is replaced by

$$
g(M) \leqslant(M-K)^{-1} \int_{a}^{M} g(t) d t .
$$


ProOF: If $g$ is nonincreasing then

$$
\frac{1}{x-K} \int_{K}^{z} g(t) d t \leqslant g(K) \leqslant \frac{1}{M-K} \int_{K}^{b} g(t) d t,
$$

that is, (6) holds. Similarly if $g$ is nondecreasing then

$$
\frac{1}{M-x} \int_{x}^{M} g(t) d t \leqslant g(M) \leqslant \frac{1}{M-K} \int_{a}^{M} g(t) d t
$$

that is, (7) holds.

Corollary 2. Let $f, g$ be positive, integrable functions on $[a, b]$ and $M, K$ real numbers satisfying $a \leqslant K<M \leqslant b$.

(i) Suppose $f$ is nonincreasing and $g$ nondecreasing. If $p>1, p^{-1}+q^{-1}=1$, then (7) holds. The inequality in (7) is reversed if $p<1$ and $f$ is nondecreasing. The equality case is as in Theorem 1(i).

(ii) Suppose $f$ is nondecreasing and $g$ nonincreasing. If $p>1, p^{-1}+q^{-1}=1$, then (9) holds. The inequality in (9) is reversed if $p<1$ and $f$ is nonincreasing. The equality case is as in Theorem 1(ii).

Proof: For (i), we have

$$
(x-K)^{-1} \int_{K}^{x} g(t) d t \leqslant(b-K)^{-1} \int_{K}^{b} g(t) d t \leqslant(M-K)^{-1} \int_{K}^{b} g(t) d t,
$$

which is true for all $x \in[K, b]$.

For (ii), we have

$$
(M-x)^{-1} \int_{x}^{M} g(t) d t \leqslant(M-a)^{-1} \int_{a}^{M} g(t) d t \leqslant(M-K)^{-1} \int_{a}^{M} g(t) d t
$$

which is true for all $x \in[a, M]$.

REMARK. Theorem A is a simple consequence of Corollary 1(i) for $b \rightarrow \infty$.

\section{REFERENCES}

[1] M. Freimer and G.S. Mudholkar, 'A class of generalizations of Hölder's inequality', in Inequalities in statistics and probability, IMS Lecture Notes, Monograph Series 5, 1984, pp. 59-67.

[2] S. Iwamoto, R.D. Tomkins and C. L. Wang, 'Inequalities and mathematical programming, III', in General inequalities 5, (W. Walter, Editor) (Basle, Boston, 1987), pp. 419-432. 
[3] D.S. Mitrinović and J.E. Pečarić, 'On the Bellman generalization of Steffensen's inequality, III', J. Math. Anal. Appl. 135 (1988), 342-345.

[4] G.S. Mudholkar, M. Freimer and P. Subbaiah, 'An extension of Hölder's inequality', J. Math. Anal. Appl. 102 (1984), 435-441.

Applied Mathematics Department The University of Adelaide

Adelaide SA 5005

Australia
Faculty of Textile Technology

University of Zagreb

Zagreb

Croatia

and

Applied Mathematics Department

The University of Adelaide

Adelaide, SA 5005

Australia 